Revue d'histoire de l'Amérique française

\title{
Contestation et continuité : les comités confessionnels et la gestion des écoles publiques au Québec (1920-1945)
}

\section{Wendy Johnston}

Volume 48, numéro 3, hiver 1995

URI : https://id.erudit.org/iderudit/305351ar

DOI : https://doi.org/10.7202/305351ar

Aller au sommaire du numéro

\section{Éditeur(s)}

Institut d'histoire de l'Amérique française

\section{ISSN}

0035-2357 (imprimé)

1492-1383 (numérique)

Découvrir la revue

\section{Citer cet article}

Johnston, W. (1995). Contestation et continuité : les comités confessionnels et la gestion des écoles publiques au Québec (1920-1945). Revue d'histoire de l'Amérique française, 48(3), 403-434. https://doi.org/10.7202/305351ar
Résumé de l'article

Le présent article porte sur la gestion de l'enseignement public au Québec entre 1920 et 1945. Il vise à jeter un nouvel éclairage sur la configuration et la nature du pouvoir scolaire central au cours de cette période marquée par des bouleversements économiques, politiques et socio-culturels, lesquels ne manquèrent pas d'avoir des répercussions sur le monde de l'éducation. Pour ce faire, il propose une analyse systématique de la composition des comités confessionnels du Conseil de l'Instruction publique et examine les luttes des différents groupes sociaux qui cherchent à améliorer la représentativité de ces importants organismes. Il en ressort que les membres des comités sont choisis, tout comme au XIX ${ }^{\mathrm{e}}$ siècle, parmi les élites cléricales, professionnelles et les milieux d'affaires. Les non-chrétiens et les représentants de la classe ouvrière en sont exclus. Quant aux femmes, les protestantes y sont sous-représentées et leurs consoeurs catholiques s'y voient refuser tout accès. Les élites franco-catholiques et anglo-protestantes tiennent à conserver un statu quo fortement marqué par des clivages entre classes sociales, groupes ethno-religieux et sexes, et ce, malgré la contestation de plusieurs groupes qui réclament un rôle dans la gestion scolaire.
Tous droits réservés @ Institut d'histoire de l'Amérique française, 1995

Ce document est protégé par la loi sur le droit d'auteur. L'utilisation des services d'Érudit (y compris la reproduction) est assujettie à sa politique d'utilisation que vous pouvez consulter en ligne.

https://apropos.erudit.org/fr/usagers/politique-dutilisation/ 


\title{
CONTESTATION ET CONTINUITÉ: LES COMITÉS CONFESSIONNELS ET LA GESTION DES ÉCOLES PUBLIQUES AU QUÉBEC (1920-1945) ${ }^{1}$
}

\author{
WENDY JOHNSTON \\ Secteur des sciences humaines \\ Université de Moncton \\ campus d'Edmundston
}

\section{RÉSUMÉ}

Le présent article porte sur la gestion de l'enseignement public au Québec entre 1920 et 1945 . Il vise à jeter un nouvel éclairage sur la configuration et la nature du pouvoir scolaire central au cours de cette période marquée par des bouleversements économiques, politiques et socio-culturels, lesquels ne manquèrent pas d'avoir des répercussions sur le monde de l'éducation. Pour ce faire, il propose une analyse systématique de la composition des comités confessionnels du Conseil de l'Instruction publique et examine les luttes des différents groupes sociaux qui cherchent à améliorer la représentativité de ces importants organismes. Il en ressort que les membres des comités sont choisis, tout comme au XIX ${ }^{e}$ siècle, parmi les élites cléricales, professionnelles et les milieux d'affaires. Les non-chrétiens et les représentants de la classe ouvrière en sont exclus. Quant aux femmes, les protestantes y sont sousreprésentées et leurs consœurs catholiques s'y voient refuser tout accès. Les élites franco-catholiques et anglo-protestantes tiennent à conserver un statu quo fortement marqué par des clivages entre classes sociales, groupes ethno-religieux et sexes, et ce, malgré la contestation de plusieurs groupes qui réclament un rôle dans la gestion scolaire.

\section{ABSTRACT}

This article deals with educational policy-making and administrative structures in Quebec's public school system from 1920 to 1945. It seeks to shed new light on the configuration and the nature of central educational power during this period characterized by economic, political and socio-cultural transformations which inevitably influenced the educational sphere. To this end, the paper undertakes a

1. Ce texte, tiré du premier chapitre de ma thèse de doctorat, L'École primaire supérieure et le high school public à Montréal de 1920 à 1945, Université de Montréal, 1991, incorpore aussi certains résultats d'une recherche post-doctorale. Mes remerciements s'adressent à René Durocher, qui a dirigé mes recherches de doctorat, à mon collègue Jacques Paul Couturier et aux évaluateurs anonymes pour leurs commentaires et suggestions. Le CRSHC et le fonds de bourses de l'Université de Montréal ont fourni une aide financière indispensable à ces recherches.

RHAF, vol. 48, no 3, hiver 1995 
systematic analysis of the composition of the confessional committees of the Council of Education and examines the struggles of the different interest groups which lobbied for better representation on these important bodies. The study demonstrates that committee members continued to be chosen, as in the $19^{\text {th }}$ century, from the ranks of the clerical, professional and business elites. Non Christians and working-class representatives were excluded from both committees, while Protestant women were under-represented and their Catholic sisters refused a place in their respective committees. Faced with challenges from groups demanding a role in educational policy-making, the Franco-Catholic and Anglo-Protestant male elites sought to preserve a status quo characterized by strong class, ethno-religious and gender biases.

Malgré l'essor de l'historiographie sur l'éducation au Québec, maints aspects des rapports entre l'école et la société avant la Révolution tranquille demeurent méconnus ${ }^{2}$. Il en va ainsi de toute la question de la gestion scolaire dans le réseau protestant et, dans une moindre mesure, dans le réseau catholique, les deux composantes du système d'enseignement public au Québec ${ }^{3}$. Il en va également ainsi, plus globalement, de l'histoire de l'éducation en langue anglaise au Québec. Les efforts historiographiques ont surtout porté sur le secteur catholique et sur les dimensions idéologiques, politiques et sociales de l'enseignement. Ainsi, Ruby Heap a examiné les relations entre l'Église catholique et l'État dans le domaine de l'enseignement public catholique durant le dernier tiers du $\mathrm{XIX}^{\mathrm{e}}$ siècle et les premières décennies du siècle suivant. Elle a notamment mis en relief l'équilibre délicat des forces en présence, de même que les tensions qui existent entre elles ${ }^{4}$. Bernard Vigod et Antonin Dupont ont repris le même

2. Sur l'état des recherches dans l'histoire de l'éducation au Canada et au Québec, on peut consulter les bilans suivants: J. Donald Wilson, «The New Diversity in Canadian Educational History», Acadiensis, 19,2 (printemps 1990): 148-169; Chad Gaffield, «Back to School: Towards a New Agenda for the History of Education», Acadiensis, 15,2 (printemps 1986): 169190; Micheline Dumont et Nadia Fahmy-Eid, «La pointe de l'iceberg: l'histoire de l'éducation et l'histoire de l'éducation des filles au Québec», Historical Studies in Education/Revue d'histoire de l'éducation, 3,2 (automne 1991): 211-236.

3. Les études qui traitent des structures administratives et décisionnelles se limitent généralement à l'examen du secteur catholique; au mieux, elles évoquent brièvement le secteur protestant. Voir L.-P. Audet, Histoire du Conseil de l'Instruction publique de la province de Québec, 1856-1964 (Montréal, Leméac, 1964); Bernard Lefebvre, L'école sous la mître (Montréal, Éditions Paulines, 1980); Pierre Boucher de La Bruère, Le Conseil de l'Instruction publique et le Comité catholique (Montréal, Le Devoir, 1918); L.-P. Audet, Histoire de l'enseignement au Québec (1608-1971), tome 2: 1840-1971 (Montréal, Holt, Rinehart \& Winston, 1971).

4. Ruby Heap, «Les relations Église-État dans le domaine de l'enseignement primaire public au Québec: 1867-1899», Société canadienne d'histoire de l'Église catholique, Sessions d'étude, 50 (1983): 183-199; et L'Église, l'État et l'enseignement primaire publique catholique au Québec, 1897-1920, thèse de Ph.D. (histoire), Université de Montréal, 1986, chapitres 1, 2, 3 et 4 . 
thème, en ce qui concerne la période où Louis-Alexandre Taschereau a été premier ministre (1920-1936) ${ }^{5}$. Dominique Jean et Thérèse Hamel, pour leur part, se sont intéressées aux débats et tensions entourant la fréquentation scolaire obligatoire durant la première moitié du $\mathrm{XX}^{\mathrm{e}}$ siècle ${ }^{6}$. Finalement, d'autres historiennes ont consacré leurs efforts à retracer la contribution des religieuses et des laïques au développement de l'enseignement privé pour les filles de religion catholique, faisant ressortir la place inférieure réservée aux élèves (filles) et aux enseignantes dans le domaine de l'enseignement public ${ }^{7}$.

Le présent article porte sur la gestion de l'enseignement public au Québec entre 1920 et 1945. Il vise à jeter un nouvel éclairage sur les formes et la nature du pouvoir scolaire central au cours de cette période marquée par des bouleversements économiques, politiques et socioculturels, lesquels ne manquent pas d'avoir des répercussions sur le monde de l'éducation. L'instruction publique est sous la responsabilité de deux comités confessionnels du Conseil de l'Instruction publique (CIP), organisme créé en 1856. À partir de 1875 et jusqu'en 1964, ce sont ces deux comités, l'un catholique, l'autre protestant, qui constituent les principales instances décisionnelles au niveau provincial. C'est en 1875, face aux pressions de certains hommes politiques conservateurs et du clergé catholique ultramontain, qui désirent renforcer le contrôle de l'institution religieuse sur l'école, que le premier ministre conservateur Boucher de Boucherville abolit le ministère de l'Instruction publique, lequel avait été mis sur pied en $1867^{8}$. $\mathrm{Au} \mathrm{XX}$ siècle, les comités confessionnels disposent toujours d'une autorité considérable dans la gestion des écoles publiques. Chacun est responsable, à l'intérieur de sa sphère de compétence, de la

5. B. L. Vigod, «Qu'on ne craigne pas l'encombrement des compétences: le gouvernement Taschereau et l'éducation, 1920-1929», Revue d'histoire de l'Amérique française, 28,2 (septembre 1974): 209-244; Quebec Before Duplessis: the Political Career of Louis-Alexandre Taschereau (Montréal et Kingston, McGill-Queen's University Press, 1986); Antonin Dupont, Les relations entre l'Église et l'État sous Louis-Alexandre Taschereau, 1920-1936 (Montréal, Guérin, 1972).

6. Dominique Jean, Familles québécoises et politiques sociales touchant les enfants, de 1940 à 1960: obligation scolaire, allocations familiales et travail juvénile, thèse de Ph.D. (histoire), Université de Montréal, 1988; Thérèse Hamel, «L'obligation scolaire au Québec: enjeu pour le mouvement syndical et agricole», Labour/Le travail, 17 (printemps 1986): 83-102; voir aussi, L'obligation scolaire au Québec: lieu et enjeu de la lutte des classes, thèse de doctorat, Université Paris V, René Descartes, 1981.

7. Voir, à titre d'exemple, les deux recueils dirigés par Nadia Fahmy-Eid et Micheline Dumont, Maîtresses de maison, maîtresses d'école: femmes, famille et éducation dans l'histoire du Québec (Montréal, Boréal Express, 1983) et Les couventines: l'éducation des filles au Québec et les congrégations religieuses enseignantes, 1840-1960 (Montréal, Boréal Express, 1986).

8. R. Heap, «Les relations Église-État...» 
réglementation pédagogique, des programmes d'études, du choix des manuels scolaires ainsi que de la formation et de la certification du personnel enseignant dans les écoles primaires et secondaires publiques. Même si plusieurs institutions scolaires privées et spécialisées échappent à leur contrôle et que les commissions scolaires locales jouent un rôle important dans la gestion quotidienne de l'enseignement public, ces comités exercent un pouvoir de première importance dans la formulation des politiques qui régissent les écoles fréquentées par la majorité des jeunes ${ }^{9}$. Les dirigeants politiques de la première moitié du $\mathrm{XX}^{\mathrm{e}}$ siècle réitèrent leur engagement à préserver ces structures. Ainsi, en 1926, le premier ministre Louis-Alexandre Taschereau rend hommage au CIP en ces termes:

Nous n'avons pas de Ministère de l'Instruction publique dans notre province. [...] Avec la grande majorité de mon parti, j'y suis hostile parce que nous avons quelque chose de mieux et de plus stable. Notre Conseil de l'Instruction publique, composé de tous les évêques de la province et d'un nombre égal de laïques [sic] dévoués et instruits offre, il me semble, des garanties qu'on pourrait peut-être nous envier ailleurs. Les ministères et les ministres passent et changent, mais ce Conseil où siègent des éducateurs avertis, hors des influences politiques, offre un caractère de permanence et de direction saine auxquelles je craindrais de substituer les nôtres ${ }^{10}$.

Le premier ministre rassure ainsi les tenants du statu quo dans l'enseignement public en s'engageant à maintenir le pouvoir scolaire à l'écart des influences politiques. Mais quelle est la véritable nature de ce conseil qui, sellon Taschereau, est «mieux» et «plus stable» qu'un ministère de l'Éducation?

Cet article propose une analyse systématique de la composition des comités confessionnels du CIP et s'attarde aux luttes des différents groupes sociaux qui cherchent à améliorer la représentativité de ces importants organismes. Nous avons divisé notre démarche en deux

9. Depuis l'abolition du ministère en 1875 , un surintendant, haut fonctionnaire nommé par le gouvernement, dirige le Département de l'Instruction publique, organisme administratif qui exécute les politiques formulées par les comités confessionnels. Le lien entre l'administration scolaire et le pouvoir politique est assuré par le secrétaire provincial, ministre du gouvernement chargé du dossier de l'éducation à l'Assemblée législative. Voir R. Heap, «Les relations ÉgliseÉtat...»; L.-P. Audet, Histoire du Conseil...

10. «Discours de M. L.-A. Taschereau, au Club de Réforme, à Montréal, le 24 avril 1926», Jules Dorion, dir., Un dossier: le discours de l'honorable M. Taschereau au Club de Réforme de Montréal, le 24 avril 1926 et les commentaires qu'il a suscités dans l'Action catholique et la presse indépendante de la Province de Québec (Québec, Action sociale Ltée, 1926), 33-34. 
parties. Dans un premier temps, nous tracerons un portrait socioprofessionnel des membres des deux comités. Dans un deuxième temps, nous examinerons les revendications qui viennent de diverses parties de la population concernant la composition de ces comités. Nous tenterons donc de cerner l'évolution de ces organismes sous l'angle de la représentativité et de mesurer la nature et l'envergure de la contestation au cours de la période choisie.

\section{I - LA COMPOSITION DES COMITÉS CONFESSIONNELS}

Pour comprendre qui sont les décideurs dans le champ éducatif à l'échelon le plus élevé, il convient d'examiner de près les caractéristiques socioprofessionnelles des membres des deux comités confessionnels. Dans la plupart des cas - soit pour $94 \%$ et $89 \%$ des membres réguliers des Comités catholique (les membres laïques) et protestant respectivement - il a été possible d'identifier leur profession ${ }^{11}$. Pour plusieurs d'entre eux, nous possédons une fiche biographique très complète.

Le Comité catholique est composé, depuis la réforme de 1875 , de tous les évêques catholiques de la province de Québec et d'un nombre égal de laïcs catholiques nommés par le lieutenant-gouverneur en conseil. Membres d'office, les évêques ont également le droit de se faire représenter par un délégué s'ils doivent s'absenter, privilège qui n'est pas accordé aux membres laïques ${ }^{12}$. La durée des mandats des membres laïques du Comité catholique n'est pas précisée dans la législation de 1875. De fait, le gouvernement considère dès la fin du $\mathrm{XIX}^{\mathrm{e}}$ siècle que ces sièges sont inamovibles ${ }^{13}$. Au même moment, la composition du CIP est remise en question par certains éducateurs. En effet, des associations d'enseignants catholiques et certains administrateurs locaux réclament aussi la nomination d'éducateurs de l'enseignement primaire ${ }^{14}$. Comme concession, à compter de 1906, le

11. Sauf indication contraire, les données pour cette analyse sont tirées des sources suivantes: Rapport du Surintendant de l'Instruction publique de la province de Québec (RSIPPQ), 1920/21-1945/46; Procès-verbaux du Comité protestant (PVCP), 1921-1947, RSIPPQ et The Educational Record of the Province of Quebec (ER); Répertoire des parlementaires québécois, 1867-1978 (Québec, Assemblée nationale du Québec, 1980); The Canadian Directory of Parliament, 1867-1967 (Ottawa, Archives publiques du Canada, 1968); Biographies canadiennes-françaises, éditions de 1920, 1922, 1923, 1924, 1925, 1926, 1928/29, 1930/31, 1931/32, 1933/34, 1934/35, 1936/37, 1942, 1948 (Montréal, Raphael Ouimet/J.-A. Fortin); B. M. Greene, ed., Who's Who in Canada, éditions de 1928/29, 1930/31, 1940/41, 1945/46 (Toronto, International Press Ltd.); Prominent People of the Province of Quebec 1923-24 (Montréal, The Biographical Society of Canada [1925]).

12. En vertu de la loi Statuts du Québec (SQ), 39 Vict. (1875), ch. 5, art. 11.

13. R. Heap, L'Église, l'État..., 118-119.

14. Ibid., 126-127. 
lieutenant-gouverneur en conseil est autorisé à nommer quatre membres adjoints provenant de l'administration scolaire: deux sont des religieux, directeurs d'écoles normales, et deux autres sont des laïcs, fonctionnaires de l'enseignement primaire. À la différence des membres réguliers, ces membres adjoints sont nommés pour un mandat n'excédant pas trois ans ${ }^{15}$.

Pour sa part, le Comité protestant compte autant de membres réguliers de religion protestante qu'il y a de laïcs au sein du Comité catholique. Ainsi, de seize en 1920, le nombre de membres réguliers du Comité protestant passe à vingt en 1945 pour suivre l'évolution du Comité catholique. Depuis la réforme de 1875, les membres du Comité protestant sont également autorisés à s'adjoindre quelques membres associés «pour les aider dans le travail de leur comité». D'abord fixé à cinq, le nombre de membres associés du Comité protestant est porté à sept en 1899, dont un, élu pour un mandat d'un an, sera le représentant de l'Association provinciale des enseignants et enseignantes protestants ${ }^{16}$.

Le poids des ecclésiastiques n'est pas le même dans les deux comités. Entre 1920 et 1945, la moitié des membres du Comité catholique sont des ecclésiastiques. Tous les évêques ou administrateurs des diocèses catholiques compris en tout ou en partie dans la province sont automatiquement nommés au Comité tout au long de cette période. De seize en 1920, le nombre d'évêques grimpe peu à peu, atteignant vingt et un en 1945. Il arrive d'ailleurs que, certaines années, le contingent laïque se retrouve légèrement minoritaire, le rythme de création de nouveaux évêchés étant plus rapide que celui de la nomination de nouveaux membres laïques. Cette forte présence des évêques au sein du Comité n'est pas sans conséquences. Certes, la plupart d'entre eux ne sont pas étrangers au domaine de l'éducation, ayant été professeurs et souvent directeurs de collèges classiques avant d'accéder à l'épiscopat, mais ils n'ont pas à affronter quotidiennement sur le terrain les problèmes de l'enseignement primaire ${ }^{17}$. Le poids des religieux est

15. $S Q, 7$ Ed. (1906), ch. 23, art. 1. Les membres adjoints n'apparaissent plus aux séances du Comité catholique après le 13 mai 1936, même si la législation qui autorise leur nomination n'est pas modifiée, peut-être parce que le comité met sur pied à cette époque des commissions permanentes faisant appel à de nombreux conseillers pédagogiques. Voir à cet effet, B. Lefebvre, L'école sous..., 31.

16. $S Q, 39$ Vict. (1875), ch. 15, art. 15; 62 Vict. (1899), ch. 28, art. 47. Le nombre de membres associés appelés au Comité protestant varie au cours de la période, allant de quatre à sept membres, mais se fixant à sept pour la plus grande partie de la période.

17. L.-P. Audet, Histoire de l'enseignement..., 2: 342; B. Lefebvre, L'école sous..., 2627. Ruby Heap abonde dans le même sens pour la période avant 1920 . Voir R. Heap, L'Église, l'État..., 130-131. 
bien moins important dans le Comité protestant. Néanmoins, de trois à cinq ecclésiastiques y siègent chaque année entre 1920 et 1945 , et le Comité est présidé par des ecclésiastiques de 1900 à 1925. Au Québec, le clergé protestant joue donc un rôle important dans la direction du réseau scolaire, bien plus longtemps qu'ailleurs au Canada, en raison de l'organisation biconfessionnelle de l'enseignement public ${ }^{18}$. Ainsi, le journal montréalais The Gazette applaudit chaleureusement à la nomination par le gouvernement du révérend Edward Leslie Pidgeon au Comité protestant en 1934, «thus preserving the tradition of clerical representation in the field of Protestant education ${ }^{19}$ ". Il n'y a toutefois pas unanimité sur la question au sein de la communauté. En effet, un groupe croissant de parents et d'éducateurs pendant ces années étudiées croient que l'éducation devrait être contrôlée par des spécialistes, et qu'un évêque ou un autre membre du clergé ne devrait pas être considéré un «expert» dans la matière en vertu de son office ${ }^{20}$.

L'autre moitié du Comité catholique, soit les membres laïques réguliers nommés par le gouvernement, est issue principalement des professions libérales, d'où proviennent d'ailleurs la plupart des chefs de file de la société québécoise de l'époque (tableau 1) ${ }^{21}$. La profession judiciaire y est nettement majoritaire. Avocats, juges et notaires constituent près de $70 \%$ des membres laïques au cours de la période étudiée. Si certains ont de l'expérience dans l'administration scolaire et peuvent mettre à profit leurs talents lors de la révision des règlements et de la législation scolaire, la plupart sont sans doute plus versés en droit qu'en pédagogie $e^{22}$. Le recrutement des membres laïques du Comité catholique se fait donc dans un cercle très restreint, les gouvernements poursuivant généralement la tradition selon laquelle les critères de sélection comprennent le niveau d'instruction et le statut socioprofessionnel. On n'y retrouve aucun représentant de la classe ouvrière, qui compose pourtant la masse de la population et dont les enfants constituent une partie importante de la clientèle des écoles gérées par le Comité. Il en va de même pour les femmes, tenues

18. Nathan H. Mair, «The Protestant Churches», Gary Caldwell et Eric Waddell, eds., The English of Quebec: from Majority to Minority Status (Québec, Institut québécois de recherche sur la culture, 1982), 216. Voir aussi à cet effet, «Loss of Religion in Schools Feared», Montreal Daily Star, $1^{\text {er }}$ avril 1938.

19. The Gazette, 29 juin 1934. 1937.

20. «Protestant Parents Oppose Church-Dominated Schools», The Gazette, 17 septembre

21. Robert Boily, «Les hommes politiques du Québec, 1867-1967», Vincent Lemieux, dir., Personnel et partis politiques au Québec: aspects historiques (Montréal, Boréal Express, 1982), 93-117.

22. B. Lefebvre, L'école sous..., 29. 
en pratique à l'écart de la gestion scolaire, même si le cadre législatif ne les exclut pas spécifiquement des comités confessionnels. Formé dans les collèges classiques et les universités, le personnel laïque représente donc, comme le note l'historien de l'éducation LouisPhilippe Audet, «l'infime minorité de la population et l'on peut se demander si elle est suffisamment en contact avec le peuple et consciente de tous ses besoins ${ }^{23}$ ».

TABLEAU 1

Profession des membres laïques du Comité catholique 1920-1945

\begin{tabular}{lcr}
\hline Profession & Nombre & $\%$ \\
\hline avocat & 12 & 34,3 \\
juge & 9 & 25,7 \\
éducateur/administrateur scolaire & 7 & 20,0 \\
notaire & 3 & 8,6 \\
médecin & 1 & 2,9 \\
journaliste & 1 & 2,9 \\
inconnue & 2 & 5,7 \\
Total & 35 & 100,0 \\
\hline
\end{tabular}

Source: voir note 11 .

En examinant de plus près les carrières des membres issus des professions libérales, on constate que plusieurs d'entre eux ne se sont pas limités à la seule pratique de leur profession. En effet, au moins la moitié des avocats sont actifs dans le monde des affaires et sont directeurs de multiples institutions financières et industrielles ${ }^{24}$. Considérons par exemple la carrière de l'avocat et député fédéral Hector Authier qui fait partie du Comité catholique dans les années 1940. Dès le début de sa cléricature, en 1905, Authier entreprend une carrière journalistique. Il fonde et dirige à partir de 1920 le premier journal du Nord-Ouest, L'Abitibi, avant d'être nommé président de la Revue moderne Inc. en 1940. Il sera également directeur de plusieurs compagnies minières et établira la Quebec Distillers Corporation à Valleyfield en 1937. On pourrait aussi citer le cas de l'avocat

23. L.-P. Audet, Histoire de l'enseignement..., 2: 342-343.

24. Sur le phénomène des «avocats-brasseurs d'affaires» et l'interpénétration des élites politiques, professionnelles et économiques, voir Paul-André Linteau, «Rapports de pouvoir et émergence d'une nouvelle élite canadienne-française à Montréal, 1880-1914», Études canadiennes/Canadian Studies, 21 (décembre 1986): 167-170; P.-A. Linteau et al., Histoire du Québec contemporain, 1: De la Confédération à la crise (Montréal, Boréal, 1989), 520, 529. 
montréalais Joseph-Léonide Perron, membre du Comité catholique de 1906 à 1930. Celui-ci est propriétaire de la Viau Biscuits Corporation et siège aux conseils d'administration de nombreuses entreprises dont ceux de la Shawinigan Water and Power Co. Ltd et de la Montreal Tramways Ltd. Il est membre de clubs sélects et s'implique dans les associations et les activités à caractère philanthropique et socioculturel.

Le profil socioprofessionnel des membres du Comité protestant est un peu plus diversifié que celui du Comité catholique, mais son caractère élitiste est similaire (tableau 2). Les représentants des professions libérales, les avocats, les juges, les médecins et les notaires y

TABLEAU 2

Profession des membres du Comité protestant 1920-1945

\begin{tabular}{lrr}
\hline Profession & Nombre & $\%$ \\
\hline Membres réguliers & & \\
avocat & 8 & 17,0 \\
gens d'affaires & 10 & 21,3 \\
ecclésiastique & 6 & 12,8 \\
éducateur/administrateur scolaire & 5 & 10,6 \\
médecin & 4 & 8,5 \\
juge & 3 & 6,4 \\
notaire & 2 & 4,3 \\
comptable & 2 & 4,3 \\
agent d'assurances & 1 & 2,1 \\
journaliste/propriétaire de journal & 1 & 2,1 \\
inconnue & 5 & 12,8 \\
Total & 47 & 100,0 \\
Membres associés & & \\
éducateur/administrateur scolaire & 16 & 59,3 \\
avocat & 1 & 3,7 \\
notaire & 1 & 3,7 \\
comptable/conseiller administratif & 1 & 3,7 \\
marchand & 1 & 3,7 \\
journaliste & 1 & 3,7 \\
inconnue & 6 & 22,2 \\
Total & 27 & 100,0 \\
\hline
\end{tabular}

Source: voir note 11 . 
composent plus du tiers du personnel. Les gens d'affaires constituent un autre groupe important: ils représentent plus de $20 \%$ du personnel du Comité, ce qui témoigne du pouvoir que détiennent ces agents économiques au sein des élites anglo-québécoises. De fait, ils exercent leur emprise dans divers secteurs de l'économie: l'industrie manufacturière, l'exploitation des ressources naturelles, les transports, la promotion foncière, les assurances et la publicité. C'est le cas, par exemple, de Milton Lewis Hersey, qui siège au Comité durant l'entredeux-guerres. Né en 1869 à Montréal, formé au Montreal High School et détenteur d'une maîtrise en chimie de l'Université McGill, Hersey est le fondateur d'une firme de chimistes et d'ingénieurs-conseils installée à Montréal et à Winnipeg. Il siège aussi aux conseils d'administration de plusieurs entreprises canadiennes. Membre de plusieurs clubs d'élite de la métropole, tel le St. James, Hersey s'implique également dans des activités philanthropiques en tant que gouverneur des hôpitaux Montreal General et Notre-Dame.

À la différence du Comité catholique, où aucune femme ne participe à la gestion scolaire, six femmes, dont quatre font carrière dans l'enseignement, sont appelées à siéger comme membres associées au Comité protestant avant 1945. Trois enseignantes des écoles publiques, dont Catherine I. MacKenzie, directrice du Montreal High School for Girls, représentent la Provincial Association of Protestant Teachers (PAPT) pour des mandats d'un an au cours des années 1920 et 1930. Quelques femmes ont des mandats plus longs. La première et, pendant plusieurs années, la seule femme au Comité protestant, Carrie Derick, siège comme membre associée entre 1919 et 1936. Depuis le tournant du siècle, Derick est professeure de botanique à l'Université McGill. Elle est connue comme la première femme nommée à un poste de professeur titulaire dans une université canadienne et pour ses activités dans les secteurs scientifique et sociopolitique, dont la lutte pour le suffrage féminin ${ }^{25}$. C'est en 1939, à la suite des revendications faites par l'Association des diplômées de l'Université McGill, que deux autres Montréalaises sont nommées au Comité. Celles-ci sont toutes les deux diplômées de McGill et militantes dans des regroupements féminins tel le Local Council of Women. Elles participent aux travaux du Comité protestant en tant que membres associées dans les années de guerre et, pour l'une d'elles, pendant plus de quinze ans

25. Pourtant, Derick ne reçoit ni l'augmentation salariale ni tous les pouvoirs et privilèges généralement associés au rang de professeur titulaire. Voir Margaret Gillett, «Carrie Derick (1862-1941) and the Chair of Botany at McGill», Marianne Gosztonyi Ainley, ed., Despite the Odds: Essays on Canadian Women and Science (Montréal, Vehicule Press, 1990), 74-87. 
encore $^{26}$. Il faut toutefois attendre 1942, après l'adoption de la loi qui permet aux femmes de devenir commissaires d'écoles, pour voir une première femme accéder au Comité protestant à titre de membre régulier $^{27}$. Madame T. P. Ross sera par ailleurs la seule femme à jouir d'un tel statut durant le reste de l'existence du comité, étant donné qu'elle n'est pas remplacée par une femme lorsqu'elle quitte sa fonction en $1957^{28}$.

Relativement peu d'éducateurs de carrière sont appelés à siéger à titre de membre régulier des deux comités confessionnels. Les membres adjoints, qui siègent au Comité catholique entre 1906 et 1936, sont, comme le stipule la loi de 1906, professeurs ou directeurs des deux grandes écoles normales de Québec et de Montréal. Les sept autres éducateurs qui siègent au Comité catholique au cours de la période étudiée sont presque tous désignés par le gouvernement Taschereau dans les années 1920 et 1930 (voir tableau 1). On ne choisit pas des enseignants des écoles primaires mais plutôt des professeurs de l'enseignement spécialisé et supérieur, de même que des administrateurs scolaires influents. Ainsi, on y retrouve, entre autres, Joseph-Philippe Labarre, haut fonctionnaire à la Commission des écoles catholiques de Montréal, et Victor Doré, président directeur général du même organisme, qui sont nommés en 1925 et 1930 respectivement. Édouard Montpetit, professeur et secrétaire général de l'Université de Montréal, ainsi que Augustin Frigon, professeur puis directeur de l'École polytechnique et membre de divers organismes à caractère scientifique et technique, y siègent également dans l'entredeux-guerres ${ }^{29}$. Il en va de même pour le Comité protestant où l'on nomme cinq éducateurs, presque tous issus du milieu universitaire. Ainsi le Comité accueille Arthur Currie en 1920 et F. Cyril James en 1940 , qui occupent tour à tour le poste de principal à l'Université $\mathrm{McGill}^{30}$.

26. Il s'agit de Marion Byers, qui siège entre 1939 et 1944, et Florence Stalker qui conserve sa place jusqu'aux années 1960. Byers remet sa démission en 1944 mais elle sera remplacée peu après par madame Roswell Thompson qui siégera de 1945 jusqu'aux années 1960. Stalker est l'une des deux femmes nommées au Comité d'enquête Hepburn sur l'enseignement protestant au Québec (1937-1938). Sur les efforts de l'Alumnae Society de l'Université McGill en tant que groupe de pression, voir Margaret Gillett, We Walked very Warily: a History of Women at McGill (Montréal, Eden Press Women's Publications, 1981), 383.

27. La législation $S Q, 6$ Geo. VI (1942) est sanctionnée le 13 mai 1942. Madame Ross siège pour la première fois au Comité protestant le 2 octobre 1942.

28. Nous n'avons pu réunir des renseignements biographiques sur madame Ross.

29. Sur les visées réformistes de ces universitaires, voir Marcel Fournier, «Édouard Montpetit et l'Université moderne, ou l'échec d'une génération», RHAF, 36,1 (juin 1982): 3-39.

30. Certains membres religieux sont également des éducateurs. Par exemple, l'ecclésiastique anglican Arthur Huffman McGreer, qui siège au Comité pendant vingt ans à compter de 1928, est également doyen de la Faculté des arts à l'Université Bishop's. 
Minoritaires parmi les membres réguliers, les éducateurs et administrateurs scolaires de carrière représentent pourtant près de $60 \%$ des membres associés du Comité protestant (tableau 2). C'est à compter de 1899 que la PAPT se voit autorisée à élire annuellement un de ses membres à titre de membre associé, privilège qu'elle réclamait depuis plus de dix ans ${ }^{31}$. Outre ces enseignants ou directeurs des écoles primaires et secondaires qui y siègent pendant un ou deux ans, on trouve quelques professeurs et administrateurs de l'Université McGill, dont les mandats sont considérablement plus longs. Ainsi, Sinclair Laird, professeur au Macdonald College et doyen du School for Teachers, est membre associé de 1913 jusqu'à la fin de la période étudiée et John Alexander Nicholson, registraire de l'université, y siège de 1921 à 1936.

En abolissant le ministère de l'Instruction publique en 1875 pour le remplacer par les comités confessionnels, le premier ministre conservateur Boucher de Boucherville visait, selon ses dires, à placer «l'enseignement primaire à l'abri des influences plus ou moins dommageables, dans une atmosphère élevée et sereine d'où ne se feraient plus sentir ni l'esprit de caste, ni les agitations des luttes politiques ${ }^{32} \gg$. Les dirigeants politiques de la première moitié du $\mathrm{XX}^{\mathrm{e}}$ siècle répètent le même refrain. Ainsi, en 1926, le premier ministre Taschereau prétend que le CIP est mieux et plus stable qu'un ministère de l'Instruction publique. Hector Perrier, le secrétaire provincial durant le régime libéral d'Adélard Godbout, se dit du même avis en 1940 lorsqu'il s'adresse à ses collègues du Comité catholique. Il «n'est jamais entré dans ma pensée, dit-il, de favoriser la création d'un ministère de l'Instruction publique. La politique est une aventure qui comporte beaucoup d'instabilité, alors que la direction générale de notre enseignement doit pouvoir s'exercer avec sérénité et esprit de continuitée ${ }^{33}$ ». Or, dans la réalité, les comités confessionnels sont loin d'être à l'abri de la politique. En effet, il ne suffit pas d'être un membre de l'élite professionnelle ou des milieux de l'éducation pour y être nommé. Encore faut-il généralement être ardent partisan ou du moins sympathisant du parti politique au pouvoir.

Les comités confessionnels sont d'autant moins à l'abri de la politique que plusieurs parlementaires en font partie. Plusieurs membres

31. Allan D. Talbot, P.A.P.T. The First Century: a History of the Provincial Association of Protestant Teachers of Quebec (Montréal, P.A.P.T., 1963), 29.

32. Propos de Boucher de Boucherville, cités dans Boucher De La Bruère, Le Conseil de l'Instruction publique..., 72.

33. Procès-verbaux du Comité catholique (PVCC), séance du 11 décembre 1940. Voir aussi à cet effet les propos du premier ministre Duplessis et du secrétaire provincial Omer Côté en 1944. PVCC, séance du 27 septembre 1944. 
de ces comités cumulent leurs responsabilités dans le champ scolaire avec celles de député provincial ou fédéral, conseiller législatif ou sénateur, perpétuant une tradition qui remonte aux débuts du CIP ${ }^{34}$. Ainsi, onze des trente-cinq membres laïques du Comité catholique sont aussi députés provinciaux et parfois ministres. On y retrouve même Lomer Gouin, qui siège au Comité catholique entre 1898 et 1929 , soit avant, durant et après son mandat comme premier ministre. Les députés fédéraux ne sont pas en reste: sept des trente-cinq membres siègent au Parlement fédéral pendant leur mandat au Comité. La majorité des membres du Comité catholique sont des libéraux, ce qui n'est guère surprenant étant donné la prédominance des administrations libérales pendant cette période. Tout comme celles qui les ont précédées, les administrations Taschereau et Godbout ne manquent pas l'occasion de nommer des amis du régime lorsque surviennent des vacances au Comité catholique ${ }^{35}$. Pour leur part, huit des quarante-six membres réguliers du Comité protestant siègent à l'Assemblée législative, tandis que cinq sont élus à la Chambre des communes, cinq sont appelés au Conseil législatif et un, au Sénat. Dans certains cas, les mêmes individus cumulent deux de ces postes politiques durant leur mandat au Comité. Soulignons enfin la présence de ministres influents au Comité protestant, notamment celle de presque tous les trésoriers de la province dans l'entre-deux-guerres.

Outre le poids des hommes politiques dans le personnel des comités, il y a un autre trait frappant, soit la longévité des mandats. Les mandats de vingt et même trente ans ne sont pas rares. On y trouve donc plusieurs membres âgés, ce qui introduit un écart parfois très grand entre ceux-ci et les familles dont les enfants sont touchés par les politiques scolaires. Ainsi, il n'y a pas que l'origine sociale qui sépare les membres des comités confessionnels de la population qu'ils desservent; dans plusieurs cas, il y a aussi des générations entières. D'ailleurs, au moins seize des trente-cinq membres laïques qui font partie du Comité catholique au cours de ces années, et dont le mandat prend fin avant 1946, sont décédés en fonction ${ }^{36}$. Les évêques catholiques, pour leur part, ont presque tous conservé leur siège jusqu'à leur mort. C'est également le cas de dix des quarante-six membres réguliers du Comité protestant dont le mandat se termine entre 1920 et $1946^{37}$. À titre d'exemple, le pasteur anglican Elson Irving Rexford

34. R. Heap, L'Église, l'État..., 121-123; B. Lefebvre, L'école sous..., 28-29.

35. R. Heap, L'Église, l'État..., 119-120.

36. Il s'agit du chiffre minimal car nous ne connaissons pas l'année du décès pour tous les membres laïques du comité.

37. Parmi les membres associés les mandats sont plus courts, les membres moins âgés et l'implication politique moins grande. Nous avons repéré un seul membre associé qui est décédé en fonction et deux qui ont un mandat politique à titre de conseiller législatif. 
siège au Comité protestant de 1892 jusqu'à son décès en 1936, à l'âge de 86 ans. De même, le mandat de l'avocat Joseph-Emery Robidoux au Comité catholique dure près de trente ans, ne prenant fin qu'avec son décès à l'âge de 86 ans en 1929.

\section{2 - LES REMISES EN QUESTION}

Du point de vue de la composition, les instances décisionnelles centrales demeurent donc relativement homogènes de 1920 à 1945 , renvoyant ainsi l'image de stabilité et de permanence de l'appareil éducatif. Doit-on pour autant conclure à l'existence d'un consensus social en matière de gestion scolaire? Ce n'est pas le cas. Plusieurs groupes d'intérêt s'agitent en effet pour réclamer la démocratisation des principaux organismes qui régissent l'école publique. Des groupes de femmes, la communauté juive, les représentants ouvriers et les associations regroupant le personnel enseignant sont parmi ceux qui revendiquent des changements au cours de ces années.

\section{Les femmes}

Ainsi, les femmes sont tenues à l'écart de la participation à la gestion scolaire centrale. Elles le sont largement dans le cas des protestantes et complètement dans le cas des catholiques. Cette exclusion n'est guère surprenante dans un contexte où les Québécoises sont privées des droits politiques aux échelons local et provincial et où la capacité légale des femmes mariées est sévèrement limitée ${ }^{38}$. Les attitudes des élites cléricales et professionnelles des milieux francophones sont fortement influencées par la conception des rapports sociaux entre les sexes préconisée par l'Église catholique. Ainsi, la femme est surtout identifiée à ses fonctions d'épouse et de mère, ce qui permet de l'écarter de maintes activités et responsabilités jugées extérieures à sa place «naturelle» dans la sphère domestique ${ }^{39}$. De

38. La législation sur le droit de vote et d'éligibilité au niveau provincial est adoptée en 1940. SQ, Geo. VI (1940), ch. 7. Les parlementaires étendent aux femmes mariées le droit de vote aux élections municipales en 1941, puis en 1942 celles-ci obtiennent le droit de vote aux élections des commissaires d'écoles (auparavant limité aux veuves et célibataires majeures). On établit aussi l'éligibilité des femmes à ces postes. $S Q$, Geo. VI (1941), ch. 41, 69; $S Q, 6 \mathrm{Geo}$. VI (1942), ch. 20. Sur la statut juridique des femmes, on peut consulter Jennifer Stoddart: «Quand des gens de robe se penchent sur les droits des femmes: le cas de la commission Dorion, 19291931», Marie Lavigne et Yolande Pinard, dir., Travailleuses et féministes: les femmes dans la société québécoise (Montréal, Boréal Express, 1983), 307-335.

39. Marie-Aimée Cliche, «Droits égaux ou influence accrue? Nature et rôle de la femme d'après les féministes chrétiennes et les antiféministes au Québec, 1896-1930», Recherches féministes, 2,2 (1989): 101-119; Collectif Clio, L'histoire des femmes au Québec depuis quatre siècles (Montréal, Le Jour, éditeur, 1992), 331-336; Jean Hamelin et Nicole Gagnon, Histoire du catholicisme québécois, dirigée par Nive Voisine, Le XXe siècle, tome 1: 1898-1940 (Montréal, 
plus, les revendications féministes sont perçues par les élites francocatholiques du début du $\mathrm{XX}^{\mathrm{e}}$ siècle comme autant de menaces aux fondements sociaux de la collectivité francophone qui vit alors dans un contexte de changement social rapide ${ }^{40}$. Même parmi les regroupements féminins catholiques, la question des droits politiques ne fait pas l'unanimité. En effet, la Fédération nationale Saint-Jean-Baptiste doit renoncer à la lutte pour le suffrage féminin à cause des pressions du clergé, tandis que l'Association des Cercles de fermières du Québec, qui regroupe des femmes rurales, adopte une position antisuffrage dans l'entre-deux-guerres ${ }^{41}$. Les élites anglo-protestantes se montrent davantage ouvertes à la participation des femmes à la vie publique, en raison peut-être de traditions socioreligieuses moins restrictives à l'égard des femmes et compte tenu de leur situation plus confortable comme groupe majoritaire en Amérique du Nord. Par ailleurs, à la différence des femmes catholiques, qui doivent composer avec les religieuses qui investissent une grande partie des postes de direction dans les champs de l'éducation, de la santé et des services sociaux, plusieurs protestantes accèdent à des postes de responsabilité dans ces domaines. Les femmes du milieu anglo-protestant ont ainsi la possibilité de travailler plus souvent en collaboration avec les hommes. Cela peut expliquer en partie le fait que les militantes angloquébécoises trouvent des appuis chez les élites masculines dans leurs luttes pour le suffrage féminin et la réforme du Code civil ${ }^{42}$.

Malgré tout, depuis le tournant du siècle, et particulièrement dans l'entre-deux-guerres, des groupes de femmes tant catholiques que protestantes revendiquent une meilleure place pour les femmes dans les organismes de direction scolaire aux niveaux local et provincial ${ }^{43}$. Cette campagne est menée en parallèle avec la lutte plus publique et plus systématique pour le suffrage féminin et l'égalité juridique des Québécoises. En 1928, dans la foulée de la réorganisation des forces

Boréal Express, 1984), 325-331; «Les femmes ne doivent pas se mêler de politique», L'enseignement primaire (EP), 43,7 (mars 1922): 456-457; «Contre le suffrage féminin», EP, 44,9 (mai 1923): 568 .

40. Susan Mann Trofimenkoff, Visions nationales: une histoire du Québec (SaintLaurent, Éditions du Trécarré, 1986), ch. 6.

41. Marie Lavigne et al., «La Fédération nationale Saint-Jean-Baptiste et les revendications féministes au début du $20^{e}$ siècle», Travailleuses et féministes..., 199-217; Yolande Cohen, Femmes de parole: l'histoire des Cercles de fermières du Québec, 1915-1990 (Montréal, Le Jour, éditeur, 1990), 245-246.

42. Voir J. Stoddart, «Quand les gens de robe...», 317-318, 334.

43. Le Montreal Local Council of Women réclame dès 1899 la nomination d'une femme à la commission scolaire protestante de la ville. Voir Catherine L. Cleverdon, The Women Suffrage Movement in Canada (Toronto, University of Toronto Press, 1974), 218, 223-224. 
féministes au Québec, un comité spécial réunissant des militantes montréalaises anglophones et francophones s'adresse au surintendant de l'Instruction publique pour demander que des femmes puissent être nommées au CIP et reconnues éligibles à siéger aux commissions scolaires. Plusieurs arguments sont présentés pour justifier la participation des femmes à la gestion scolaire: le fait qu'elles composent la moitié des élèves et la majorité du corps enseignant, qu'elles jouent déjà un rôle bénéfique en tant que directrices des écoles de filles et administratrices scolaires ailleurs au Canada et à l'étranger et, surtout, la notion que les femmes sont «par vocation» les éducatrices naturelles des jeunes ${ }^{44}$. Par ailleurs, l'intérêt et les affinités particulières des femmes dans le domaine de l'éducation sont souvent cités comme un argument militant en faveur de la participation politique féminine au niveau provincial ${ }^{45}$.

Sur cette question, les autorités scolaires catholiques et protestantes affichent, comme on peut s'y attendre, des attitudes divergentes. En effet, le Comité protestant s'est déclaré favorable à la représentation féminine dans les instances décisionnelles dès 1916 et accueille lui-même quelques femmes comme membres associées depuis $1918^{46}$. Le Comité catholique, quant à lui, refuse catégoriquement de faire une place aux femmes et rejette la requête présentée par le comité spécial. Sans explication aucune, le Comité catholique qualifie la demande de «non opportune ${ }^{47}$ ». En revanche, à la même époque, le Comité catholique félicite la Ligue catholique féminine pour sa campagne contre «l'immodestie des vêtements ${ }^{48}$ ». Invitées à collaborer à certaines activités à caractère éducatif, les femmes catholiques se voient pourtant refuser une place parmi les décideurs dans le champ scolaire.

Lorsque plusieurs groupes anglo-protestants contestent à la fin des années 1930 la composition du Comité protestant, des groupes tant masculins que féminins, tel le Montreal Board of Trade, se prononcent en faveur de la représentation des femmes au sein des commissions

44. ANQQ, E 13, Fonds du ministère de l'Éducation, Correspondance générale (CG), Lettres reçues (LR), article 1706, pièce 150 (1928), Eva R. Thibaudeau et Madeleine Sheridan à C. Delâge, 8 et 29 janvier 1928.

45. Voir, à titre d'exemple, Idola Saint-Jean, «L'aristocratie des sexes», Le monde ouvrier, 28 janvier 1928.

46. Voir les procès-verbaux des séances du Comité protestant du 25 février 1916 et du 24 février 1928; ANQQ, E 13, CG, LR, article 1706, pièce 150 (1928), secrétaire du Comité protestant à Madeleine Sheridan, 7 mars 1928.

47. PVCC, séances du $1^{\text {er }}$ février et du 9 mai 1928; ANQQ, E 13, CG, LR, article 1706, pièce 150 (1928), Cyrille Delâge à Eva R. Thibaudeau, 13 février et 15 mai 1928.

48. $P V C C$, séance du $1^{\text {er }}$ février 1928. 
scolaires et du Comité protestant ${ }^{49}$. Du côté catholique, l'obtention du suffrage féminin en 1940 et la présence au pouvoir du gouvernement libéral d'Adélard Godbout encouragent les féministes à entreprendre une nouvelle campagne afin d'obtenir la représentation des femmes au sein du Comité catholique. Ainsi, Idola Saint-Jean, présidente de l'Alliance canadienne des électrices, et Émilia L. Lamarche, secrétaire du même regroupement, s'adressent au premier ministre Godbout en décembre 1941 pour demander la nomination d'une femme au Conseil du travail et au Comité catholique. Notant «la bienveillante sympathie» manifestée par Godbout et son cabinet envers la cause des femmes, et qualifiant l'adoption du suffrage féminin de «geste magnifique», ces militantes avouent leur étonnement devant le fait qu'aucune représentante n'ait été nommée au sein de ces deux organismes. Saint-Jean et Lamarche prennent parti pour les enseignantes et font remarquer qu'il en va de l'intérêt de ces dernières de nommer une femme laïque au Comité catholique, car «une femme laïque comprendrait mieux les questions qui intéressent les institutrices ${ }^{50} \gg$. Pour sa part, Thérèse Casgrain, qui dirige la Ligue pour les droits de la femme, y va à son tour en janvier 1942 d'une demande pour la nomination des femmes au Comité catholique. Elle insiste sur le rôle d'éducatrice des mères, sur l'importance des femmes dans le système scolaire en tant qu'enseignantes et étudiantes, en ajoutant que deux femmes siègent déjà au Comité protestant ${ }^{51}$.

À la différence du Comité catholique en 1928, le premier ministre Godbout ne rejette pas d'emblée ces demandes. Il promet au contraire que son gouvernement y prêtera attention ${ }^{52}$. Pourquoi le gouvernement ne donne-t-il pas suite à ces requêtes? Les sources consultées ne font pas écho des débats qui ont pu avoir lieu au Comité catholique ou dans les coulisses du pouvoir. Mais la présence imposante des évêques au Comité catholique y est sans doute pour quelque chose. Rappelons que le premier ministre Godbout n'a réussi à faire taire l'opposition cléricale à son projet de loi sur le suffrage féminin qu'à coup de menaces. Godbout a dû aviser le cardinal Villeneuve, porte-parole des évêques, qu'il démissionnerait et serait remplacé par le ministre T. D. Bou-

49. Voir, par exemple, ANQQ, E 13, CG, LR, J. S. Cook, secrétaire du MBOT à J. H. A. Paquette, secrétaire provincial, 21 février 1939 et «Highlights of the Brief», Montreal Daily Star, 18 février 1938; "Recommendations of the Department of Education and Better Schools, Quebec Women's Institute», Home and Country (mai 1938).

50. ANQQ, E 13, CG, LR, article 2217, pièce 77 (1942), Idola Saint-Jean et Émilia L. Lamarche à A. Godbout, 30 décembre 1941.

51. Ibid., Thérèse F. Casgrain à A. Godbout, 16 janvier 1942.

52. Ibid., A. Godbout à I. Saint-Jean, 7 janvier 1942; lbid., A. Godbout à madame PierreF. Casgrain, 27 janvier 1942. 
chard, dont les vues anticléricales étaient bien connues ${ }^{53}$. L'inaction du gouvernement s'explique peut-être aussi par la forte présence de membres du Barreau au sein du Comité catholique. Le Barreau refusera d'autoriser l'admission des femmes en son sein jusqu'en 1941. De nombreux parlementaires considèrent que l'entrée des femmes au Barreau serait un premier pas vers l'accession des femmes à un rôle politique, car la profession d'avocat débouche souvent sur l'action politique ${ }^{54}$.

Même l'adoption de la législation permettant aux femmes de devenir commissaires d'écoles en 1942 n'ouvre pas la voie aux femmes catholiques dans la gestion scolaire centrale. Le Comité catholique n'emboîte pas le pas au Comité protestant qui nomme une première femme comme membre régulier en 1942. En effet, aucune femme n'est appelée à siéger au Comité catholique avant son abolition dans les années 1960. Ainsi, l'obtention des droits politiques formels n'est pas suffisante pour garantir aux femmes l'accès à la représentation politique, et encore moins au pouvoir ${ }^{55}$.

Cette opposition féroce à la participation féminine du côté catholique est pourtant paradoxale dans la mesure où elle va à l'encontre du discours qui prévaut chez les élites masculines de l'époque. Thérèse Casgrain souligne les contradictions qui marquent la position de ces dernières, car «la femme de chez nous appelée avec force envolées de rhétorique l'éducatrice par excellence, est privée de toute autorité dès qu'il s'agit du problème de l'éducation ${ }^{56}{ }^{\prime}$. Autre paradoxe frappant: qu'en est-il du principe, maintes fois réitéré par les chefs de file masculins, que les comités confessionnels sont à l'abri de la politique? Comment peut-on alors justifier l'exclusion des femmes d'un tel organisme «non politique»?

53. Collectif Clio, L'histoire des femmes du Québec, 364.

54. Par contre, la médecine et la comptabilité s'ouvrent aux femmes en 1930. Voir Sylvie Bélanger, «Thérèse Casgrain et l'entrée des femmes au Barreau», Anita Caron et al., dir., Thérèse Casgrain: une femme tenace et engagée (Sainte-Foy, Presses de l'Université du Québec, 1993), 105-106.

55. Dès l'automne 1942, plusieurs protestantes sont élues commissaires d'écoles au Québec. En 1945, on nomme la première femme à la commission scolaire protestante de Montréal. Une première femme est nommée à la commission scolaire catholique de la métropole à la fin des années 1960. Voir PVCP, séance du 27 novembre 1942 dans $E R, 59,2$ (avril-juin 1943): 123; Thérèse F. Casgrain, Une femme chez les hommes (Montréal, Éditions du Jour, 1971), 275.

56. Thérèse Casgrain, «La femme a-t-elle justice dans Québec?», séance de l'Agora, le 12 mars 1939, dans Quelques problèmes d'actualité dans le Québec (Montréal, Éditions Beauchemin, 1939), 153-154. 


\section{La classe ouvrière}

Tant du côté protestant que du côté catholique, les représentants de la classe ouvrière se voient aussi exclus des comités confessionnels. Le recrutement par voie de nomination désavantage particulièrement les classes populaires qui se font également refuser une place dans les commissions scolaires montréalaises. Cette exclusion des lieux du pouvoir scolaire n'est pas acceptée par tous. Ainsi, des représentants des syndicats dits «internationaux», qui regroupent la majorité des travailleurs syndiqués, réclament depuis le début du siècle l'élection des commissaires d'écoles catholiques à Montréal ${ }^{57}$. Certains groupes contestent aussi le manque de représentativité des comités confessionnels. Dans le but d'y remédier, l'Association catholique de la jeunesse canadienne-française se prononce à la veille de la Première Guerre mondiale en faveur de l'élection, par les parents, de la plupart des membres laïques du Comité catholique ${ }^{58}$.

$\mathrm{Ce}$ sont les organisations ouvrières, regroupant surtout des travailleurs qualifiés, qui canalisent l'élan réformiste des classes populaires en matière d'enseignement public, particulièrement du côté catholique. Or, il existe de profondes divisions idéologiques à ce sujet au sein du mouvement ouvrier québécois. En effet, tout comme les groupes de femmes, les organisations ouvrières ne présentent pas un front uni sur les questions scolaires. Les syndicats internationaux ont longtemps favorisé la création d'un ministère de l'Éducation afin que les décideurs de l'enseignement public soient directement responsables devant le peuple. Ils ont également reclamé depuis le début du siècle l'instauration de la gratuité scolaire et de la fréquentation scolaire obligatoire, afin d'améliorer l'accès à l'enseignement et le taux de scolarisation ${ }^{59}$. Par contre, les syndicats confessionnels regroupés

57. Voir, à titre d'exemple, le témoignage de Georges Raoul Brunet, vice-président de l'Exécutif provincial du Conseil des métiers et du travail devant la Commission d'enquête sur la situation des écoles catholiques de Montréal de 1926-1927 dans ANQQ, E 26-11, article 32, pièce 273 , dépositions XI, 8 octobre 1926, 34-49. Notons que malgré la croissance de syndicats catholiques à l'époque, les syndicats affiliés à la Fédération américaine du travail regroupent environ $60 \%$ des syndicats québécois entre 1916 et 1931. Ceux-ci représentent encore $44 \%$ des syndicats québécois en 1940. Voir Jacques Rouillard, Histoire du syndicalisme au Québec (Montréal, Boréal, 1989), 129-131, 157, 210.

58. ACJC, Étude critique de notre système scolaire (Montréal, Bureaux de l'ACJC, 1913), 47-48.

59. Memoire du parti ouvrier à la Commission royale d'enquête sur l'éducation, dans Le Pays, 14 mai 1911, reproduit dans Groupe de chercheurs de l'Université du Québec à Montréal sur l'histoire des travailleurs québécois, L'action politique des ouvriers québécois (fin du XIX siècle à 1919). Recueil de documents (Montréal, Presses de l'Université du Québec, 1976), 65; «Discours de M. L.-A. Taschereau, au Club de Réforme, à Montréal, le 24 avril 1926», 33-34; Thérèse Hamel, «L'obligation scolaire au Québec». 
au sein de la Confédération des travailleurs catholiques du Canada, à la remorque des autorités cléricales, s'opposent à tout ce qui est perçu comme une ingérence de l'État dans l'éducation ${ }^{60}$.

Des clivages ethniques empêchent également le mouvement ouvrier de parler d'une seule voix et d'entreprendre des actions concertées afin d'obtenir la représentation des travailleurs dans les instances chargées de la gestion scolaire. Anglophones et francophones agissent séparément. Depuis le début du siècle, des ouvriers francophones regroupés dans des syndicats de métiers et appuyant le Parti ouvrier à Montréal dénoncent le fait qu'aucun des leurs ne se retrouve au Comité catholique. Ainsi, en 1911, Pierre T. Masson s'adresse au nom des ouvriers montréalais au rédacteur du journal montréalais Le Pays pour rappeler les besoins de ce groupe en matière d'éducation, soit «des livres moins chers, l'admission gratuite aux écoles, le droit d'élire nos commissaires» et l'obtention de «un ou deux sièges au Conseil de l'Instruction publique ${ }^{61}{ } \gg$. Ce n'est que lorsque les ouvriers siégeront aux comités confessionnels que ses autres membres, laïques ou ecclésiastiques, pourront «savoir ce que ça coûte aux pauvres gens de faire instruire leurs enfants, avec le système actuel ${ }^{62}{ }^{2}$. Les organisations ouvrières ne cessent de remettre en question le caractère élitiste du Comité catholique. Un éditorial de janvier 1939 dans Le monde ouvrier, organe officieux des syndicats internationaux au Québec, critique la décision du Comité catholique de prohiber l'enseignement de l'anglais avant la $5^{\mathrm{e}}$ année, concluant qu'une telle décision, qui va à l'encontre des intérêts des ouvriers, est attribuable à ce manque de représentation: «Mais au fait, les ouvriers sont-ils représentés sur ce corps important? Évidemment non, mais pourquoi pas ${ }^{63}$ ?»

Les interventions d'organisations syndicales à prédominance anglophone au sujet de la gestion scolaire semblent plus rares. Une seule organisation ouvrière qui regroupe des travailleurs anglophones, soit la Fraternité canadienne des cheminots, présente un mémoire au comité d'enquête Hepburn à la fin des années $1930^{64}$. En 1938,

60. Ibid.; Jacques Rouillard, Les syndicats nationaux au Québec de 1900 à 1930 (Québec, Presses de l'Université Laval, 1979), 217.

61. Pierre T. Masson, ouvrier à monsieur le rédacteur, 12 janvier 1911, Le Pays, 14 janvier 1911, reproduit dans L'action politique des ouvriers..., 67-68.

62. Ibid., 68.

63. «Pourquoi nous devons apprendre l'anglais», Le Monde ouvrier/The Labour World, 14 janvier 1939.

64. Voir Protestant Education in the Province of Quebec: Report of the Quebec Protestant Education Survey, (s.l., décembre 1938), appendix I (A) et (B). Nous n'avons pu localiser ce mémoire. 
A. L. Hepworth, le secrétaire de cette importante organisation ouvrière, s'adresse à Hepburn pour lui rappeler que «no mention has been made of the inclusion of a representative of the organized working population of the province» au sein du Comité protestant et ce, malgré le fait que la majorité des électeurs viennent des foyers ouvriers ${ }^{65}$. Hepworth propose donc un candidat mais sa requête, même si elle est acheminée par Hepburn à W. P. Percival, le directeur provincial de l'enseignement protestant, demeure lettre morte ${ }^{66}$. Deux ans plus tard, Hepworth revient à la charge pour revendiquer de nouveau la nomination d'un représentant ouvrier au Comité protestant. Cette fois-ci, Percival répond en affirmant qu'il n'existe pas alors de vacance au comité, tout en référant la requête de Hepworth au lieutenant-gouverneur qui est responsable des nominations ${ }^{67}$.

Il n'est pas facile de cerner les attitudes des autorités protestantes et catholiques concernant la représentation ouvrière au sein des instances administratives supérieures. Néanmoins, certaines prises de position sur la question du mode de recrutement des commissaires d'écoles montréalais sont révélatrices de la vision partagée par plusieurs membres des élites cléricales et laïques. À la fin des années 1930, le Comité protestant s'oppose avec véhémence à la proposition de combler les sièges à la commission scolaire par voie d'élection plutôt que par nomination. Ce système en vigueur dans la métropole depuis 1846 est préférable, selon le Comité protestant, car «the men of the class who have served the Protestant Board for many years will not, it has been proven, offer themselves for competitive election with all its unpleasantness ${ }^{68} 》$. Cette même notion que la gestion scolaire

65. ANQQ, E 13, CG, LR, article 2055, pièce 1675 (1937), Dossier: «Inquiries concerning Educational system relative to proposed survey (Première partie)», A. L. Hepworth à Hepburn, 11 avril 1938. Indépendante du syndicalisme international, la Fraternité canadienne des cheminots/Canadian Brotherhood of Railway Employees, s'unit en 1927 à la Fédération canadienne du travail pour fonder la nouvelle centrale syndicale, le Congrès pancanadien du travail. En 1936, il y a au Québec 31 syndicats affiliés à la Fraternité. Voir Rouillard, Histoire du syndicalisme..., 130-131, 163-164.

66. Le candidat proposé est George J. McClure de Ville LaSalle. Nous n'avons pas trouvé copie de la réponse ni de Hepburn ni de Percival à cette requête.

67. ANQQ, E 13, CG, LR, article 2055, pièce 1675 (1937), Dossier: «Requests for Survey Reports (8 $8^{\text {ième }}$ partie)», Percival à A. L. Hepworth, 24 janvier 1940; Percival à George J. McClure, 13 avril 1940; Percival à A. L. Hepworth, 21 mai 1940.

68. Ibid., Dossier: «Miscellaneous material concerning Quebec Protestant Education Survey Committee», «First draft, January 1939 - Statement of facts presented by the Protestant Committee of the Council of Education concerning the Report of the Quebec Protestant Education Survey that was presented on December 16, 1938», 24. Voir aussi à cet effet, Statement concerning the Report of the Quebec Protestant Education Survey presented by the Protestant Committee of the Council of Education (Québec, 1939), 25-27. 
devrait rester entre les mains d'une élite éclairée se retrouve dans les milieux franco-catholiques ${ }^{69}$.

\section{Les non-chrétiens}

La question de la place faite aux non-chrétiens dans le système scolaire public se pose avec acuité dans le cas des Juifs de Montréal dans l'entre-deux-guerres. Selon l'Acte de l'Amérique du Nord britannique de 1867, seuls les catholiques et protestants ont des droits scolaires spécifiquement reconnus. Or, l'importance croissante de la communauté juive dans la métropole depuis le tournant du siècle et son statut ambigu dans les écoles publiques protestantes, où environ un tiers de la clientèle est d'origine juive, mènent à un état de crise dans les années $1920^{70}$. En effet, les parents juifs sont tenus à l'écart de la direction scolaire tant locale que centrale même s'ils payent leurs taxes scolaires à la commission scolaire protestante et que leurs enfants fréquentent ses écoles ${ }^{71}$. La communauté juive est cependant divisée sur la question de la gestion scolaire. D'une part, un groupe assimilationniste cherche l'égalité des droits à l'intérieur du réseau scolaire protestant. D'autre part, un groupe autonomiste réclame plutôt la création d'un troisième réseau scolaire confessionnel. Ces divergences idéologiques se doublent de clivages socioéconomiques. En effet, les partisans des écoles juives séparées se recrutent parmi les immigrants récents, les rabbins orthodoxes et les organisations ouvrières, tandis que les adeptes d'une participation égale dans le système existant se trouvent parmi les uptowners, généralement des

69. Voir, par exemple, l'échange entre $\mathrm{M}^{\mathrm{gr}}$ Deschamps et George Raoul Brunet lors de la Commission d'enquête sur la situation des écoles catholiques de Montréal de 1926-1927, dans ANQQ, E 26-11, article 32, pièce 273, dépositions XI, 8 octobre 1926, 40.

70. Voir Pierre Anctil, Le rendez-vous manqué: les Juifs de Montréal face au Québec de l'entre-deux-guerres (Québec, Institut québécois de recherche sur la culture, 1988), 165-209; B. L. Vigod, Quebec Before Duplessis..., 89, 156-161; L.-P. Audet, Histoire de l'enseignement..., 238-247; A. Dupont, Les relations..., 253-273.

71. Cette situation existe depuis la fin du $X X^{e}$ siècle, grâce à une entente entre la communauté juive et la commission scolaire protestante. Une loi de 1903 sanctionne cet état de choses en assimilant les Juifs aux protestants quant à l'enseignement public à Montréal. $S Q$, 3 Ed. VII (1903), ch. 16. Notons que la commission scolaire protestante impose également des limites sur l'embauche des enseignants juifs. La communauté juive revendique à plusieurs reprises depuis le début du siècle, mais sans succès, le droit de faire élire ou faire nommer des représentants à la commission scolaire protestante de Montréal. P. Anctil, Le rendez-vous..., 176177; Leon D. Crestohl, The Jewish School Problem in the Province of Quebec (Montreal, Eagle Publishing Co., Ltd., 1926), 5-9; Archives nationales du Canada (ANC), Papiers Lomer Gouin, MG 27, III B 4, Mémoires, notes et journaux, vol. 53, microfilm, bobine C-2359, «Commission appointed to study and report on school system of the Island of Montreal: Minutes of proceedings under section B: the education of Jewish children in Protestant or other schools», 30 septembre - 2 octobre 1924, 30208-30213. 
individus mieux nantis, profondément canadianisés et réformistes ou semi-orthodoxes sur le plan religieux ${ }^{72}$.

Les différences d'opinion au sein de la communauté juive font surface lors de la commission d'enquête nommée en 1924 par le premier ministre Taschereau pour régler la question du statut des Juifs dans le système scolaire. Présidée par l'ancien premier ministre Lomer Gouin, la commission comprend trois commissaires protestants et trois commissaires juifs. Ceux-ci sont divisés. Deux commissaires adoptent une position assimilationniste, réclamant les mêmes droits que les protestants dans le système scolaire existant; le troisième favorise plutôt la séparation scolaire. Quant aux commissaires protestants, ils favorisent le statu quo, tout en cherchant une compensation financière pour l'instruction des enfants juifs ${ }^{73}$. La commission d'enquête, qui remet son rapport au gouvernement en décembre 1924, ne réussit donc pas à trouver une solution acceptable pour les parties en cause. Un seul point fait l'unanimité parmi les commissaires: la nécessité d'obtenir un jugement des tribunaux sur la constitutionnalité d'un partage du pouvoir scolaire avec les Juifs.

Les tribunaux sont donc appelés à trancher. La plus haute instance judiciaire du pays, le Comité judiciaire du Conseil privé, rend en février 1928 une décision qui semble favoriser les tenants du statu $q u o$. Selon le tribunal, la législation de 1903, qui sanctionnait l'entente entre la communauté juive et la commission scolaire protestante de Montréal, n'accorde aux Juifs que le droit de fréquenter les écoles protestantes et ne leur permet pas de siéger ni à la commission scolaire ni au Comité protestant. Le gouvernement provincial peut cependant choisir de créer une commission scolaire confessionnelle juive sur le modèle des commissions scolaires protestantes et catholiques, dans la mesure où un tel système ne porte pas atteinte aux droits et privilèges traditionnels des catholiques et des protestants ${ }^{74}$. Le débat se poursuit

72. Sur l'évolution des courants d'opinion au sein de la communauté juive, on consultera avec profit P. Anctil, Le rendez-vous..., 165-209.

73. B. L. Vigod, Quebec Before Duplessis..., 156-158; ANC, MG 27, «Commission appointed to study...»; Québec, Rapport de la Commission spéciale d'éducation (Québec, Imprimeur du Roi, 1925). Notons que dans les années 1930, Arthur Currie et E. W. Beatty, qui siègent à cette commission, appliquent des politiques d'admission défavorables aux étudiants juifs à l'Université McGill. Voir P. Anctil, Le rendez-vous..., 181, 209, note 10. Trois commissaires catholiques, qui étudient d'autres questions touchant l'enseignement catholique sur l'île de Montréal, font également partie de la Commission spéciale. Ces derniers endossent le rapport des commissaires protestants.

74. Hirsch et Cohen v. The Protestant Board of School Commissioners of the City of Montreal and the Protestant Committee of the Council of Public Instruction in the Province of Quebec, 1928, A.C., 200-216. 
jusqu'à la fin de 1928 et en 1929. Le gouvernement y va même de propositions visant à accorder aux Juifs une place au sein du CIP. Pourtant, la communauté juive demeure divisée et le statu quo persiste.

Les revendications de ceux qui ne sont ni catholiques ni protestants et qui veulent participer directement à la gestion scolaire se heurtent à l'opposition farouche des élites des deux principales croyances religieuses. La réaction des autorités scolaires protestantes à la possibilité de voir des Juifs faire leur entrée dans le Comité protestant est viscérale. Aux yeux de la plupart des membres du Comité protestant, une telle proposition constitue une injustice qui va à l'encontre de la convention historique qui accorde à la minorité protestante du Québec l'autonomie complète dans le domaine de l'enseignement. Lors d'une réunion spéciale du Comité protestant au début de janvier 1930, les membres votent donc massivement pour communiquer au gouvernement leur «strong disapproval of any attempt to deprive the Protestant minority of their exclusive control of Protestant education in this Province ${ }^{75}{ }_{\gg}$. Seulement deux des dix-sept membres présents enregistrent des votes dissidents, soit Carrie Derick, la seule femme siégeant comme membre régulier, et Adam Kirk Cameron, un homme d'affaires montréalais. Tous deux soulignent l'injustice dont sont victimes les non-protestants, qui sont taxés mais qui n'ont pas le droit d'être représentés ${ }^{76}$. Quelques jours plus tard, le révérend Elson I. Rexford, membre du Comité protestant, s'adresse au rédacteur du Montreal Daily Star pour expliquer la position majoritaire du comité. La nomination des Juifs serait, selon Rexford, «the beginning of a disorganizing influence in the Protestant educational system of the Province» et les membres du comité «feel that they have no mandate from the Protestant population of the Province to surrender sacred rights which Protestants have enjoyed in this province for more than half a century». L'enjeu est fondamental:

The legislation suggested is no mere side issue. It strikes at the keystone of our Protestant educational system. The creation of the Protestant Committee with its powers and duties has always been regarded as the guarantee of our Protestant educational liberties in the province. ${ }^{77}$

75. ANQQ, E13, CG, LR, article 1760, pièce 25 (1930), «Extract from the Minutes of the proceedings of a Special Meeting of the Protestant Committee of the Council of Education held in Montreal on Friday the 3rd day of January 1930».

76. «Protestant Body Opposes Change», Montreal Daily Star, 3 janvier 1930.

77. Montreal Daily Star, 8 janvier 1930. Rexford a également publié, quelques années auparavant, un ouvrage qui exprime son hostilité face à la présence des Juifs dans les écoles publiques. Voir Our Educational Problem: the Jewish Population and the Protestant Schools (Montréal, Renouf, 1924). 
Sa collègue Carrie Derick ne partage pas ce point de vue. Dans une lettre adressée au même journal, elle élabore sa pensée et ose suggérer que les écoles protestantes publiques soient transformées en écoles neutres:

It seems to me that duty to the community as a whole should outweigh «rights» secured for a group of citizens, when conditions were not the same as now. I recognize that the establishment of a common school system is impossible in the Province of Quebec. But I think that amendments to existing laws should be made which would permit the conversion of Protestant into non-Catholic schools, in relation to which all non-Catholic citizens would be equal. If it were desired to supplement ethical teaching by dogmatic «religious instruction», the latter could be given by experts to children of their respective faiths, in hours set aside for the purpose ${ }^{78}$.

Mais le point de vue de Derick représente une voix dans le désert. En effet, la plupart des autorités protestantes sont solidement ancrées dans le camp de l'opposition. Il en va ainsi des commissaires scolaires protestants de la ville de Montréal, qui font savoir au premier ministre Taschereau qu'une telle réforme «would be an infringement of the rights of the Protestant minority in this province to direct and control their own schools ${ }^{79}{ }^{\prime}$. La détermination des anglo-protestants à conserver intégralement les droits constitutionnels et religieux consentis au groupe est par ailleurs appuyée par les évêques catholiques. Craignant que d'autres minorités non chrétiennes réclament leur propre secteur scolaire et que cela ne conduise à la création d'un système scolaire unique et neutre sur le plan religieux, l'épiscopat catholique entreprend une campagne de pressions auprès du gouvernement au début de $1930^{80}$. Dans leur défense acharnée du statu quo biconfessionnel, les élites anglo-protestantes se montrent donc les meilleures alliées de l'Église catholique au Québec. La minorité anglo-protestante tient non moins jalousement que l'épiscopat catholique au maintien de ses prérogatives dans le champ scolaire, afin de s'assurer le contrôle de ses écoles ${ }^{81}$.

78. Montreal Daily Star, 11 janvier 1930. Schools, (Montréal, Renouf, 1924).

79. Archives de la Commission des écoles protestantes du Grand Montréal (ACEPGM), «Minutes Montreal Protestant Central School Board», vol. II, séance du 14 janvier 1930; ANQQ, E 13, CG, LR, article 1760, pièce 25 (1930), James Smyth, Président du Protestant Board of School Commissioners of the City of Montreal à L.-A. Taschereau, 10 janvier 1930.

80. $\mathrm{M}^{\mathrm{gr}}$ Georges Gauthier, «Projet de commission scolaire juive», Semaine religieuse de Montréal, 89,12 (1930): 179-186; L.-P. Audet, Histoire de l'enseignement..., 243-244.

81. Un éditorial dans l'organe officiel du secteur protestant du Département de l'Instruction publique, The Educational Record, se réjouit ainsi lorsque le secrétaire anglophone du DIP est nommé directeur de l'enseignement protestant au Québec: «the title signifies [...] how 
L'agitation tant protestante que catholique en faveur de l'exclusion des Juifs du Conseil de l'Instruction publique porte fruit. En effet, la crise se dénoue au début de 1931 sans que la situation de la communauté juive ne soit guère meilleure qu'au début du siècle. Le seul droit que les Juifs se voient confirmer demeure celui, pour leurs enfants, de fréquenter les écoles protestantes de la métropole. Les autorités scolaires protestantes de Montréal, pour leur part, obtiennent de nouvelles dispositions financières qui allègent le fardeau financier représenté par la présence des enfants juifs dans leurs écoles ${ }^{82}$. Ainsi, le calme se rétablit à propos de cette question controversée, même si l'on n'a pas répondu aux aspirations de la minorité juive. Par ailleurs, avant la fin des années 1930, le Comité protestant doit faire face à une contestation généralisée, alors que plusieurs secteurs de la société remettent en question les bases du recrutement de cet organisme.

\section{Le comité d'enquête Hepburn et le Comité protestant}

Vers la fin des années 1930, plusieurs composantes de la minorité anglo-protestante se livrent à une remise en question d'envergure des structures administratives supérieures dans le champ scolaire. Des indices d'insatisfaction s'accumulent dans ce contexte de crise économique et le Comité protestant, jugé peu représentatif et inefficace, devient la cible de critiques ${ }^{83}$. En septembre 1937, tous les membres du Comité protestant sont priés de démissionner à la demande des cinq députés protestants de l'Assemblée législative; ces derniers font état des revendications de citoyens de toutes les parties de la province et de tous les milieux depuis l'été 1936 et qui réclament des réformes radicales de l'enseignement et la réorganisation du comité ${ }^{84}$. Les circonstances entourant cette demande demeurent un peu obscures; elle ouvre néanmoins la voie à un débat public désiré depuis longtemps. Les appels à la réforme lancés au même moment par Adam Kirk Cameron et Carrie Derick ne sont pas appuyés par la

completely Protestant education in the Province is and has been under Protestant control, through the Department and the Protestant Committee of the Council of Public Instruction». «Director of Protestant Education», ER, 44,4-5 (avril-juin 1924): 97-98. Voir aussi W. P. Percival, «Protestant Education in Province of Quebec», Quebec Chronicle-Telegraph, 8 octobre 1936.

82. En vertu de la loi $S Q, 21$ Geo. V (1931), ch. 63, art. 10.

83. Montreal Junior Board of Trade, Report of the Committee on the Protestant School Situation in Montreal (Montréal, décembre 1936), 36-37; «Education here Meets Criticism», Montreal Star, 29 novembre 1935; «Changes Sought in School Board», Montreal Star, 12 juin 1936.

84. «Seven are Ousted by Minister», Montreal Herald, 10 septembre 1937; «Education Committee are all Asked to Resign», Montreal Star, 14 septembre 1937; «Protestant Committee Protest Reorganization», Montreal Star, 24 septembre 1937; «Will Our School System Get Good Marks?», The Standard, 12 mars 1938. 
majorité des membres du Comité protestant. Les membres du Comité contestent cette intervention des députés protestants en soulignant que cela constitue un précédent dangereux et qu'aucune explication ne leur est par ailleurs fournie. Le premier ministre Duplessis confirme le statu quo en octobre 1937, une décision qui est applaudie par plusieurs membres de la communauté anglo-protestante. La remise en cause du Comité protestant ne cesse pas pour autant. Plusieurs groupes d'intérêt, des institutions scolaires et des associations professionnelles réclament même la mise sur pied d'une commission d'enquête sur l'enseignement protestant. Parmi eux, on compte les membres protestants de l'Assemblée législative et du Conseil législatif, la PAPT, la Provincial Association of Protestant School Boards (qui représente les commissions scolaires en dehors des grands centres urbains), l'Université McGill, le Montreal Protestant Central School Board, le Montreal Board of Trade et divers groupes de femmes ${ }^{85}$.

Face à de telles pressions, le Comité protestant décide en novembre 1937 de mettre sur pied un comité d'enquête pour examiner tous les aspects du système scolaire protestant à l'échelle du Québec. Indice du souci de protéger l'autonomie du réseau scolaire protestant, ce comité relève non pas du gouvernement mais plutôt du Comité protestant lui-même. On fait appel à un expert de l'extérieur, soit le fonctionnaire écossais de l'éducation, W. A. F. Hepburn, pour présider le survey. Outre son président, le comité se compose d'une dizaine de membres, dont deux femmes, provenant des milieux de l'éducation et de la philanthropie, de l'Église anglicane, des professions libérales et du monde des affaires. Le Comité protestant confie au comité Hepburn un vaste mandat, soit d'enquêter et de faire rapport «on all matters affecting Protestant education in the Province of Quebec ${ }^{86} »$.

Les travaux du comité se terminent à la fin de mai 1938. Hepburn est mandaté par les membres pour rédiger le rapport. Déposé en décembre 1938, celui-ci recommande de revoir les structures supé-

85. «Protestant School Board Reinstated by Duplessis», The Gazette, 13 octobre 1937 et «Keep Education Free from Politics», The Gazette, 19 octobre 1937. Pour les critiques, voir «Reorganization Plans for Protestant Education», Quebec Chronicle-Telegraph, 20 septembre 1937; «New Protestant Education Committee May Consist of 15 Educationists, Nine Others», Quebec Chronicle-Telegraph, 20 septembre 1937; «I.O.D.E. Interested in Education Body», The Gazette, 21 septembre 1937. "The Purpose of the Survey», The Teachers' Magazine, 20,90 (février 1938): 16-20; «Royal Commission Probe of Protestant Education Urged by McGill Leaders», The Gazette, 20 septembre 1937; ACEPGM, «Minutes PBSCCM», vol. 20, séance du 23 septembre 1937.

86. Le comité Hepburn reçoit quelque 120 documents écrits et tient 49 réunions, dont 11 à l'extérieur de Montréal. Voir Protestant Education..., vii-xi et appendices. 
rieures de l'administration scolaire, en particulier la composition et les fonctions du Comité protestant. En effet, selon le rapport, «few things in the course of their investigation impressed the members of the Survey Committee more than the range and depths of the criticisms directed against the policy and administration of the Protestant Committee ${ }^{87}$ ». Ce dernier «had, for the time being, lost the confidence of many thoughtful members of the Protestant community in every part of the Province ${ }^{88}{ }$. On recommande donc que le Comite protestant «as at present constituted should be dissolved», et que le nouveau comité réorganisé soit davantage représentatif des divers groupes d'intérêt, des organismes scolaires, des deux sexes et des différentes régions du Québec ${ }^{89}$.

Hepburn recommande que les membres réguliers soient toujours nommés par le lieutenant-gouverneur en conseil, en nombre égal au personnel laïque du Comité catholique, mais avec des mandats d'une durée maximale de quatre ans. De plus, ce personnel comprendrait des membres désignés par l'Association provinciale des commissions scolaires protestantes, la Commission scolaire protestante du Grand Montréal, la PAPT, les recteurs des universités Bishop's et McGill, des pasteurs protestants, des femmes ainsi que des députés protestants. Selon cette répartition, les représentants des milieux de l'éducation seraient majoritaires, occupant dix des dix-sept sièges réguliers. Ce nouveau comité choisirait également dix membres associés, dont trois femmes, et il inviterait quelques administrateurs scolaires et universitaires à servir comme conseillers. Ces modifications tiennent compte des reproches les plus fréquemment mis de l'avant au sujet du Comité protestant et sont fidèles à l'esprit des propositions faites par la PAPT dans son mémoire ${ }^{90}$. Elles témoignent par ailleurs de la montée des éducateurs comme groupe professionnel qui revendique une place de choix dans la gestion scolaire.

Quel accueil la minorité anglo-protestante réserve-t-elle à ces recommandations? Il n'est guère surprenant que le Comité protestant s'oppose au projet de réorganisation, qui le vise directement. À ses yeux, les réformes proposées s'attaquent au mode traditionnel de recrutement et mettent en danger les acquis de la minorité. La plupart

87. Protestant Education..., 17-18.

88. Ibid.

89. Ibid., 267.

90. Ibid., 267-274; Brief submitted to the Quebec Protestant Education Survey by the PAPT of Quebec with which is associated the High School Principals' Association (mars 1938): 4-10; voir aussi les demandes pour une meilleure représentation des milieux ruraux présentée dans le mémoire du Provincial Association of Protestant School Boards, «Rural School Boards Submit Brief», Montreal Daily Star, 22 février 1938. 
des membres du Comité protestant sont indignés: «It cannot be expected [...] that the Protestant Committee will make a recommendation concerning its own abolition. This is not because of a selfish desire to remain in office but simply because it considers that it is responsible to the people for the conduct of Protestant education in the Province and finds it amazing that a body appointed by itself and paid for with funds that it has obtained from the Government should bring in such a recommendation ${ }^{91}$.» Par ailleurs, W. P. Percival, le directeur de l'enseignement protestant, publie en 1940 un ouvrage, Life in School, qui présente un portrait très flatteur du système scolaire protestant au Québec. Si certains éducateurs et journalistes au Québec en font l'éloge, le qualifiant de plus juste et plus utile que le rapport Hepburn, d'autres observateurs à l'extérieur du Québec le voient comme une réponse complaisante à ce dernier ${ }^{92}$. Il est clair que Percival cherche depuis l'époque de l'enquête Hepburn à refaire la crédibilité du Comité protestant. Il se réjouit donc de voir qu'aucun de ses membres ne démissionne face aux pressions publiques et que, lorsque survient une vacance parmi les membres réguliers au début de 1940, on a recours au processus traditionnel de sélection, ce qui est interprété par certains journalistes comme une justification du Comité protestant ${ }^{93}$.

Par contre, la structure proposée dans le rapport Hepburn semble plutôt chaleureusement reçue par les organismes et particuliers reliés à l'éducation ainsi que par certains membres du public. Le rapport suscite un grand intérêt à sa sortie et, dès le début de 1939, les autorités publiques sont inondées de lettres venant de groupes qui récla-

91. ANQQ, E 13, CG, LR, article 2055, pièce 1675 (1937), Dossier «Miscellaneous material concerning Quebec Protestant Education Survey Committee», «First draft, January 1939 - Statement of facts presented by the Protestant Committee of the Council of Education concerning the Report of the Quebec Protestant Education Survey that was presented on December 16, 1938", 97. Voir aussi Statement concerning the Report of the Quebec Protestant Survey presented by the Protestant Committee of the Council of Education (Québec, 1939), 1222. Il existe au moins une voix dissidente parmi les membres du Comité protestant. W. $O$. Rothney, professeur de pédagogie au Bishop's University, nommé membre régulier au Comité protestant en 1928, se prononce publiquement en faveur de la reconstitution du Comité en 1939. Voir sa lettre «An Explanation», Montreal Daily Star, 22 mars 1939.

92. Les réactions à l'ouvrage de Percival, Life in School: an Explanation of the Protestant School System of the Province of Quebec (Montréal, The Herald Press, 1940) se trouvent dans ANQQ, E 13, Director of Protestant Education, article 3013, spicilège concernant «Life in School, 1940-1942».

93. ANQQ, E 13, CG, LR, article 2055, pièce 1675 (1937), Dossier: «Requests for Survey Reports $\left(8^{\text {ième }}\right.$ partie)«, W. P. Percival à C. L. Sibley, editor, Montreal Daily Herald, 21 décembre 1938. Voir aussi les lettres de Percival à plusieurs ministres, dont Henri Groulx et J. A. Mathewson, datées 23, 27 janvier 1940, avec copie de l'éditorial «The Vindication of the Protestant Committee of Education», Montreal Daily Herald, 17 janvier 1940 dans Ibid. 
ment la mise en vigueur des recommandations qu'il contient ${ }^{94}$. Pourtant, les recommandations du rapport Hepburn sur ce sujet vont demeurer en plan au cours de la période étudiée, ce qui n'est pas sans soulever le mécontentement de certains groupes. Ainsi, lorsque le gouvernement Godbout entreprend une vaste enquête sur la fréquentation scolaire en 1942, l'Association provinciale des enseignants protestants demande si «the findings of this survey will result in any more action than did the Hepburn Report ${ }^{95}$ ». Soucieux de l'autonomie des protestants dans le champ éducatif, le gouvernement n'osera pas aller à l'encontre des vœux du Comité protestant pour reconstituer cet organisme.

Par contre, la collectivité catholique ne s'engage pas à la même époque dans une telle remise en question de la représentativité de ses gestionnaires scolaires. Certes, au début des années 1940, dans un climat plus favorable à la réforme sociale, l'association qui représente les enseignants et les enseignantes laïques francophones de Montréal réclame une représentation plus importante des leurs au Comité catholique $^{96}$. Mais la contestation du personnel enseignant, de même que les appels des organisations ouvrières et de certains libéraux radicaux pour un ministère de l'Éducation, ne trouvent pas d'appuis généralisés dans la population. Lorsque le Comité catholique s'interroge sur son rôle vers la fin des années 1930, ce n'est pas pour remettre en question sa composition, ni l'étendue de son contrôle, mais plutôt pour consolider et élargir ses pouvoirs dans le champ scolaire. C'est une réforme de l'intérieur et non une consultation populaire ou un débat public qu'entame le Comité catholique à l'automne $1936^{97}$.

\section{CONCLUSION}

Cette étude des comités confessionnels lève donc le voile sur le visage du pouvoir scolaire central et des enjeux qui l'entourent dans le Québec de l'entre-deux-guerres et du début des années 1940, non

94. Voir la correspondance dans ANQQ, E 13, CG, LR, articles 2054 et 2055, pièce 1675 (1937), Dossiers: «Survey: $4^{\text {ìme }}$ partie»; «Inquiries... ( $5^{\text {ième }}$ partie)»; «Requests for Survey Reports ( $8^{\text {ième }}$ partie)». Plusieurs journaux réservent également un accueil favorable au rapport Hepburn: voir à titre d'exemple, «Educational Survey: an Important Document», Montreal Daily Star, 19 décembre 1938; «The Education Report», Sherbrooke Daily Record, 17 décembre 1938.

95. «Education Survey is much Criticized», The Gazette, 28 mai 1942. Voir aussi Miriam Chapin, «War Conditions Aggravate Faults in School System», Montreal Standard, 24 juillet 1943.

96. Alliance catholique des professeurs de Montréal, Les réformes de l'enseignement primaire (Montréal, 1941), 14.

97. PVCC, séance de septembre 1936. 
seulement dans le secteur catholique, dont les contours nous sont plus familiers, mais aussi dans le secteur protestant, moins connu. Le pouvoir scolaire ne constitue, à plusieurs égards, qu'un autre volet du pouvoir politique. Le principe cher aux élites professionnelles et cléricales d'une direction scolaire à l'abri de la politique est un mythe. L'analyse de la composition des comités confessionnels révèle non seulement le poids des députés parmi les membres, mais aussi le caractère politique des nominations faites au sein des deux organismes. En 1945, tout comme au XIX ${ }^{\mathrm{e}}$ siècle, le personnel des Comités protestant et catholique se recrute parmi les élites cléricales et professionnelles et dans les milieux d'affaires. Les non-chrétiens et les représentants de la classe ouvrière sont écartés de la gestion scolaire tout au long de la période, tant par les chefs de file anglo-protestants que franco-catholiques. Quant aux femmes de confession protestante, elles se voient accordée une place, si limitée soit-elle, au Comité protestant, mais aucune de leurs consœurs catholiques n'est appelée à siéger au Comité catholique. Même après que les femmes eurent été conviées à participer au processus électoral au niveau provincial, municipal et dans les commissions scolaires locales, les milieux masculins catholiques tiennent à les éloigner des lieux d'exercice du pouvoir dans l'enseignement public.

La composition des instances administratives supérieures ne subit donc pas de transformations majeures entre 1920 et 1945 , et ce, malgré les mutations sociales et idéologiques associées au développement de l'économie et à la progression de l'urbanisation. Plusieurs groupes dénoncent le caractère peu représentatif des comités confessionnels. La critique est particulièrement forte dans les communautés anglo-saxonne et juive, comme le démontrent la crise des écoles juives et les débats entourant les travaux du comité d'enquête Hepburn. Mais les forces en présence résistent à une nouvelle répartition des pouvoirs entre les autorités religieuses, l'État et la population. Le spectre de l'État dans l'école continue de hanter les esprits du côté catholique mais trouble aussi la minorité anglo-protestante. Les membres du clergé, l'épiscopat catholique et la plupart des chefs de file des deux communautés réagissent avec véhémence face à toute suggestion qui menace de porter atteinte aux pouvoirs des comités confessionnels et au caractère confessionnel des écoles. Ils se transforment en chiens de garde pour préserver le statu quo biconfessionel établi au XIX ${ }^{\mathrm{e}}$ siècle contre les prétentions de ceux qui ne sont ni catholiques ni protestants. De plus, si la participation des femmes est davantage tolérée dans les milieux anglo-protestants que du côté catholique, les élites des deux collectivités veulent maintenir le contrôle de l'appareil scolaire entre les mains de la bourgeoisie. 
Cette exploration de la gestion scolaire, qui met en relief la diversité et la complexité des intérêts présents dans la société québécoise entre 1920 et 1945, suggère aussi la richesse de ce domaine de recherche et l'étendue du terrain qui reste à défricher. Plusieurs autres pistes mériteraient d'être étudiées à fond. Il serait instructif, par exemple, de se pencher sur le rôle des protestantes et des catholiques dans l'administration scolaire locale après 1942. La configuration du pouvoir scolaire de l'entre-deux-guerres, qui s'inscrit dans une continuité qui remonte au $\mathrm{XIX}^{\mathrm{e}}$ siècle, laisse également des traces profondes dans le système scolaire public québécois de l'après-guerre. L'héritage des structures confessionnelles résiste même aux réformes des années 1960 et, trente ans plus tard, sert encore d'obstacle à la réorganisation de l'administration de l'enseignement primaire et secondaire sur des bases linguistiques. 